\title{
Genetic obesity: next-generation sequencing results of 1230 patients with obesity
}

\author{
Lotte Kleinendorst, ${ }^{1}$ Maarten P G Massink, ${ }^{2}$ Mellody I Cooiman, ${ }^{3}$ Mesut Savas, ${ }^{4}$ \\ Olga $\mathrm{H}$ van der Baan-Slootweg, ${ }^{5}$ Roosje J Roelants, ${ }^{6}$ Ignace C M Janssen, ${ }^{3}$ \\ Hanne J Meijers-Heijboer, ${ }^{1,7}$ Nine V A M Knoers, ${ }^{2}$ Hans Kristian Ploos van Amstel, ${ }^{2}$ \\ Elisabeth F C van Rossum, ${ }^{4}$ Erica L T van den Akker, ${ }^{8}$ Gijs van Haaften, ${ }^{2}$ \\ Bert van der $Z_{\text {waag, }}{ }^{2}$ Mieke M van Haelst ${ }^{1,7}$
}

- Additional material is published online only. To view, please visit the journal online (http://dx.doi.org/10.1136/ jmedgenet-2018-105315).

For numbered affiliations see end of article.

\section{Correspondence to} Dr Mieke M van Haelst, Department of Clinical Genetics VU Medical Center Amsterdam The Netherlands; m.vanhaelst@vumc.nl

Received 6 February 2018 Revised 21 May 2018 Accepted 10 June 2018 Published Online First 3 July 2018

\section{ABSTRACT}

Background Obesity is a global and severe health problem. Due to genetic heterogeneity, the identification of genetic defects in patients with obesity can be time consuming and costly. Therefore, we developed a custom diagnostic targeted next-generation sequencing (NGS)based analysis to simultaneously identify mutations in 52 obesity-related genes. The aim of this study was to assess the diagnostic yield of this approach in patients with suspected genetic obesity.

Methods DNA of 1230 patients with obesity (median BMl adults $43.6 \mathrm{~kg} / \mathrm{m}^{2}$; median body mass indexSD children +3.4 SD) was analysed in the genome diagnostics section of the Department of Genetics of the UMC Utrecht (The Netherlands) by targeted analysis of 52 obesity-related genes.

Results In 48 patients pathogenic mutations confirming the clinical diagnosis were detected. The majority of these were observed in the MC4R gene (18/48). In an additional 67 patients a probable pathogenic mutation was identified, necessitating further analysis to confirm the clinical relevance.

Conclusions NGS-based gene panel analysis in patients with obesity led to a definitive diagnosis of a genetic obesity disorder in 3.9\% of obese probands, and a possible diagnosis in an additional $5.4 \%$ of obese probands. The highest yield was achieved in a selected paediatric subgroup, establishing a definitive diagnosis in 12 out of 164 children with severe early onset obesity (7.3\%). These findings give a realistic insight in the diagnostic yield of genetic testing for patients with obesity and could help these patients to receive (future) personalised treatment.

\section{INTRODUCTION}

Obesity is a universal, severe health problem, with globally over 650 million adults with obesity and 124 million children and adolescents with obesity (aged 5-19 years) in 2016. ${ }^{1}$ Because of their excessive accumulation of body fat, they are at risk for many health problems, such as cardiovascular disease, type 2 diabetes mellitus, depression and certain types of cancers (eg, breast cancer and colon cancer). ${ }^{1}$ An adult is considered obese in case of a body mass index (BMI) $>30 \mathrm{~kg} / \mathrm{m}^{2} .{ }^{1}$ For from the BMI in gender and age-matched children. Obesity is caused by an imbalance between energy intake and expenditure. Environmental factors, for example, the easy accessibility of high caloric food, little physical activity or the use of obesogenic medication (eg, atypical antipsychotics or glucocorticoids), ${ }^{2}$ can severely affect this energy balance. Therefore, obesity is regarded as a multifactorial disorder. On the other hand, meta-analysis of twin and family studies have shown that the heritability of BMI is around $46 \%-72 \% .^{3}$

A number of genetic factors have indeed been identified that cause obesity. ${ }^{4}$ Nevertheless, these identified genes and chromosomal abnormalities have thus far only explained $7 \%$ of the heritability shown by twin studies. ${ }^{5}$ This percentage, however, varies depending on the country or region where the genetic studies are performed. Reports from Pakistan and Guadeloupe show a much higher prevalence of rare monogenic forms of obesity $(30 \%$ and $15 \%$, respectively). ${ }^{6} 7$ Different hypotheses have been suggested to explain the 'missing heritability' of human obesity, including CNVs, epigenetic events and rare highly penetrant variants. ${ }^{8}$

A genetic diagnosis is of great importance for patients since genetic counselling and (future) personalised therapy depending on the underlying gene defect can be offered. ${ }^{9-11}$

Additionally, a genetic diagnosis or insight in the genetic contribution to obesity might help to reduce the psychological burden of obesity, since the public distress and social stigma of being obese is a major problem for many patients with therapy-resistant obesity. $^{12}$

Due to genetic heterogeneity, the identification of genetic defects in patients with obesity can be time consuming and costly. Therefore, we developed a next-generation sequencing (NGS) gene panel analysis for patients with suspected genetic obesity and offered it in our DNA diagnostics section. For the design of our gene panel (in 2012), we selected genes associated with an obesity phenotype from the OMIM catalogue, genes associated with obesity in Genome-Wide Association Studies, in obesity or diabetes pathways (Kyoto Encyclopaedia of Genes children, BMI-SD scores (SDS) are used to define obesity (>2.3 SDS), representing the deviation and Genomes pathway database) and several genes from known obesity CNVs. With this new test, 52 obesity-related genes are simultaneously analysed.

Massink MPG, Cooiman MI

et al. J Med Genet 
Box 1 Inclusion criteria for the next-generation

sequencing obesity gene panel

Patients should (apart from the obesity phenotype) have at least one of the criteria to be included in this study.

Principal inclusion criteria:

- Age of onset of obesity $<5$ years (prepubertal onset in adult subgroups)

- Family history of obesity (alarm symptom: single person with obesity in family)

- Hyperphagia

- Intellectual deficit/developmental delay

- Congenital malformations

- Visual impairment and/or deafness

- Abnormal growth parameters (head circumference and height)

Inclusion criteria for patients undergoing bariatric surgery:

- Extreme obesity (body mass index $>50 \mathrm{~kg} / \mathrm{m}^{2}$ )

- Repeat surgery after weight regain or insufficient weight loss

The gene panel includes genes involved in both syndromic and non-syndromic monogenic obesity. Genetic variants associated with polygenic forms of obesity and obesity-associated epigenetic variants have also been described in literature, but they are not the focus of this study. ${ }^{13}$

Monogenic syndromic obesity is defined as a genetic condition caused by a single gene defect in which the patient is obese, and has additional problems, like intellectual deficit, congenital malformations, dysmorphic features and/or organ dysfunction. Monogenic non-syndromic obesity is not accompanied by intellectual deficit in the majority of cases and is often caused by mutations in the leptin-melanocortin pathway, influencing energy expenditure and food intake. ${ }^{13}$ Early onset of obesity, hyperphagia and a positive family history are often seen as warning signals for genetic non-syndromic obesity. ${ }^{14}$

\section{METHODS}

\section{Patients}

For this study, we reviewed the results of the diagnostic obesity gene panel analyses from December 2014 until April 2016. In this period, DNA samples of 1230 patients were analysed. Because of the diagnostic setting, the test was not performed on normal weight controls. The patients for which gene testing was requested, derived from 36 centres in The Netherlands and Dutch Caribbean, and two other European medical centres (from the UK and Finland). All patients/parents/guardians agreed to perform the diagnostic test and to the anonymous use of the test data. All patients were informed of their test result by the doctor who ordered the test or a genetic counsellor. Inclusion criteria to select eligible patients for the NGS obesity panel are listed in box 1. Patients who were already diagnosed with a genetic obesity disorder in the past were not included in this study.

Genetic consultations and phenotyping (figure 1) were routinely offered in five Dutch medical centres (more details are provided in the online supplementary appendix). We tried to obtain phenotypical information from the patients who were not referred for genetic consultations from the physicians who requested the test.

The median age of the total cohort was 33 years (range 0-79 years). The median age of the paediatric group was 9.5 years and of the adult group 43 years. Three hundred ninety-three patients

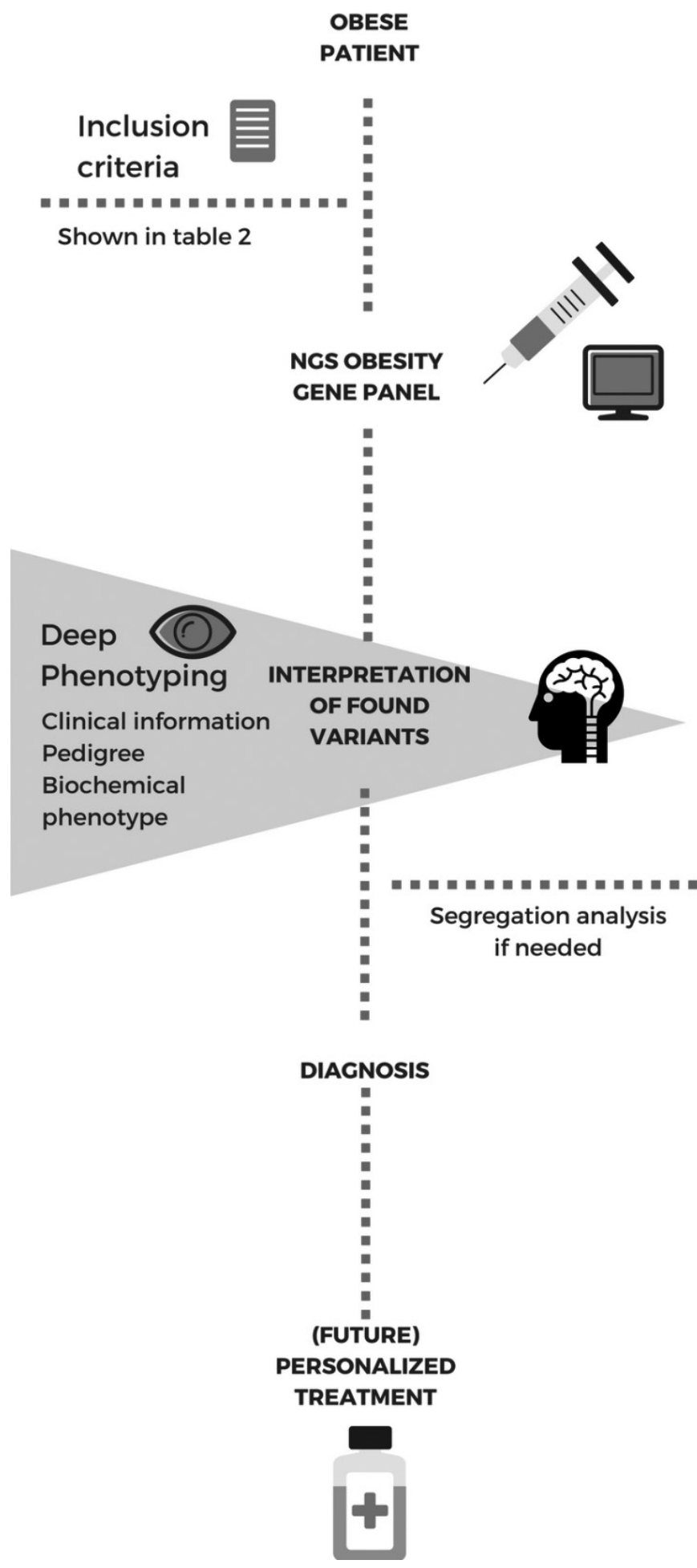

Figure 1 Diagnostic process. Patients with obesity who have one or more of the inclusion criteria can be tested with the next-generation sequencing (NGS) Obesity Gene Panel. We advised genetic counselling for all patients with abnormal results identified by the gene panel. Deep phenotyping (including pedigree information, biochemical tests and clinical dysmorphic evaluation) is needed to interpret the found variants. Sometimes, segregation analysis in the family is performed to interpret the significance of the found variant.

were younger than 18 years when the test was performed; 837 patients were older than 18 years. The median BMI of the adult patients at the time of testing was $43.6 \mathrm{~kg} / \mathrm{m}^{2}$ (lowest 22, 
highest 91). The median BMI-SD of the children was +3.4 SD (lowest $+1 \mathrm{SD}$, highest $+9 \mathrm{SD})$. The few patients with a normal BMI were all obese or morbidly obese in the past, but lost weight before testing.

\section{Patient subgroups}

For analysis of the different patient groups (eg, children with early onset obesity or patients undergoing bariatric surgery), five subgroups were created in the Dutch medical centres where genetic consultations and phenotyping were routinely offered. Our largest patient subgroup is the bariatric surgery group of 659 patients. More details about the subgroups can be found in the online supplementary appendix.

\section{Sequencing and bioinformatics analysis}

Genomic DNA was isolated from peripheral blood samples at the ISO15189 accredited Genome Diagnostics section of the Department of Genetics, UMC Utrecht (The Netherlands). Subsequently, sequencing libraries were prepared from sheared genomic DNA. Each patient and thus each sequencing library received a unique barcode consisting of 10 nucleotides. This system allows for a cost-effective and time-effective approach for batches of $\sim 50$ patients simultaneously in a single enrichment procedure. The prepared libraries were pooled and target DNA capture was performed using a custom-designed Agilent SureSelectXT assay (elid\#0561501).

The diagnostic genes included in the obesity gene panel are: ALMS1, ARL6, BBS1, BBS2, BBS4, BBS5, BBS7, BBS9, BBS10, BBS12, BDNF, CCDC28B, CEP290, CRHR2, FLOT1, G6PC, GNAS, IRS1, IRS2, IRS4, KIDINS220, LEP, LEPR, LZTFL1, MAGEL2, MC3R, MC4R, MCHR1, MKKS, MKRN3, MKS1, MRAP2, NDN, NTRK2, PAX6, PCK1, PCSK1, PHF6, POMC, PRKAR1A, PTEN, SIM1, SNRPD2, SNRPN, SPG11, TBX3, THRB, TMEM67, TRIM32, TTC8, TUB, and WDPCP. Sequencing was performed on a SOLiD 5500XL system (Life Technologies). We sequenced to an average depth of $\sim 100 \mathrm{X}$ horizontal coverage to allow for optimal variant calling. Sanger sequencing of the fourth exon of $P O M C$ was performed to obtain $>99 \%$ coverage for this gene.

\section{Variant selection}

Variant filtering and interpretation of clinical relevance

Filtering of variants was performed using the Cartagenia BENCHlab NGS module (V.3.1.2), with a validated 'classification tree'. The sequence data were compared with the dbSNP, GoNL (Genome of the Netherlands database), our in-house and Exome Variant databases (6500 exomes) to exclude common variants and select genes that contain non-synonymous variants, nonsense mutations, essential splice site mutations or coding frame-shift indels. Variants with (possible) clinical relevance were subsequently analysed in the Alamut mutation interpretation software program (V.2.6.0) using among others Polyphen2, SIFT, GERP and Grantham scores, and multiple splice-site prediction programs. The remaining (probable) pathogenic mutations were confirmed by Sanger sequencing. When the combined data were inconclusive, the variants were classified as variants of uncertain clinical significance (VUS).

\section{Statistical analysis}

Group comparisons were performed by means of the independent samples t-test. Statistical analyses were performed using SPSS software V.24.0.0.1. A Mann-Whitney U test was run to determine if there were differences in BMI in adults between those without a diagnosis and with a definite diagnosis, and in children between those with and without a definite diagnosis.

A permutation test was performed on the data of the BardetBiedl associated genes. We determined the population allele frequencies for a set of 27 curated pathogenic BBS gene mutations in our cohort. We determined the significance of this result by permutation testing on the obesity gene panel and ExAC NFE populations allele frequency data (details provided in the online supplementary appendix 1).

\section{RESULTS}

\section{Diagnostic yield}

We established a definitive diagnosis of a genetic obesity disorder in 48 patients (3.9\%), shown in tables 1 and 2, with the highest yield in a paediatric subgroup 12/164 (7.3\%). A definitive diagnosis was established in $2.7 \%$ of the patients in the adult subgroup. Six of the 48 patients $(12.5 \%)$ had pathogenic mutation that causes syndromic obesity. The majority of the identified mutations however, are linked to non-syndromic obesity. In 67 additional patients (5.4\%), VUS were found that could possibly lead to a future diagnosis (see online supplementary table S1). Seventeen variants in comorbidity genes were identified (see online supplementary table S2). Eleven out of 52 genes in the panel harboured pathogenic mutations confirming the diagnosis; 44 genes showed (probable) pathogenic mutations or VUS.

\section{BMI in patients with a genetic obesity disorder}

The median BMI in adult patients with a definitive diagnosis was $41.8 \mathrm{~kg} / \mathrm{m}^{2}$ (range 34.2-72.7). Patients without a definitive or likely diagnosis had a median BMI of $43.7 \mathrm{~kg} / \mathrm{m}^{2}$ (range 22.4-91). Median BMI was not statistically significantly different between the two groups (details in the online supplementary appendix). The median BMI-SD in children with a definitive diagnosis was +3.84 (corrected for age and gender). In children without a definitive or likely diagnosis, the median BMI-SD was +3.4 (corrected for age and gender). This was also not a statistically significant difference (online supplementary appendix).

\section{Carrier status}

61 patients (5\% of the total cohort) were identified as carriers of a heterozygous known pathogenic mutation that only leads to an obesity phenotype in an autosomal recessive mode of inheritance (ALMS1, PCK1, SPG11, TUB, BBS genes and modifiers). These findings were assessed as non-relevant for the development of the obesity phenotype, but patients were counselled about these results because the findings could impact the health of future generations or reproduction decisions. An additional 76 patients $(6.2 \%$ of the total cohort) were carriers of a VUS in one of those genes. Most of them were carriers of a Bardet-Biedl syndrome (BBS)-related variant.

\section{Bardet-Biedl syndrome}

BBS is an autosomal recessive and genetically heterogeneous ciliopathy disorder characterised by obesity, intellectual deficit, retinitis pigmentosa, kidney dysfunction and polydactyly. Whether heterozygous carriers of $B B S$ genes are predisposed to obesity or not was unclear at the onset of our study. ${ }^{15} 16$

We see a 1.7-fold higher population allele frequency for $B B S$ mutation carriers in the obesity gene panel cohort compared with the ExAC's Non-Finnish European (NFE) population (see online supplementary table S3). Our permutation test showed that the permutation score was not statistically 


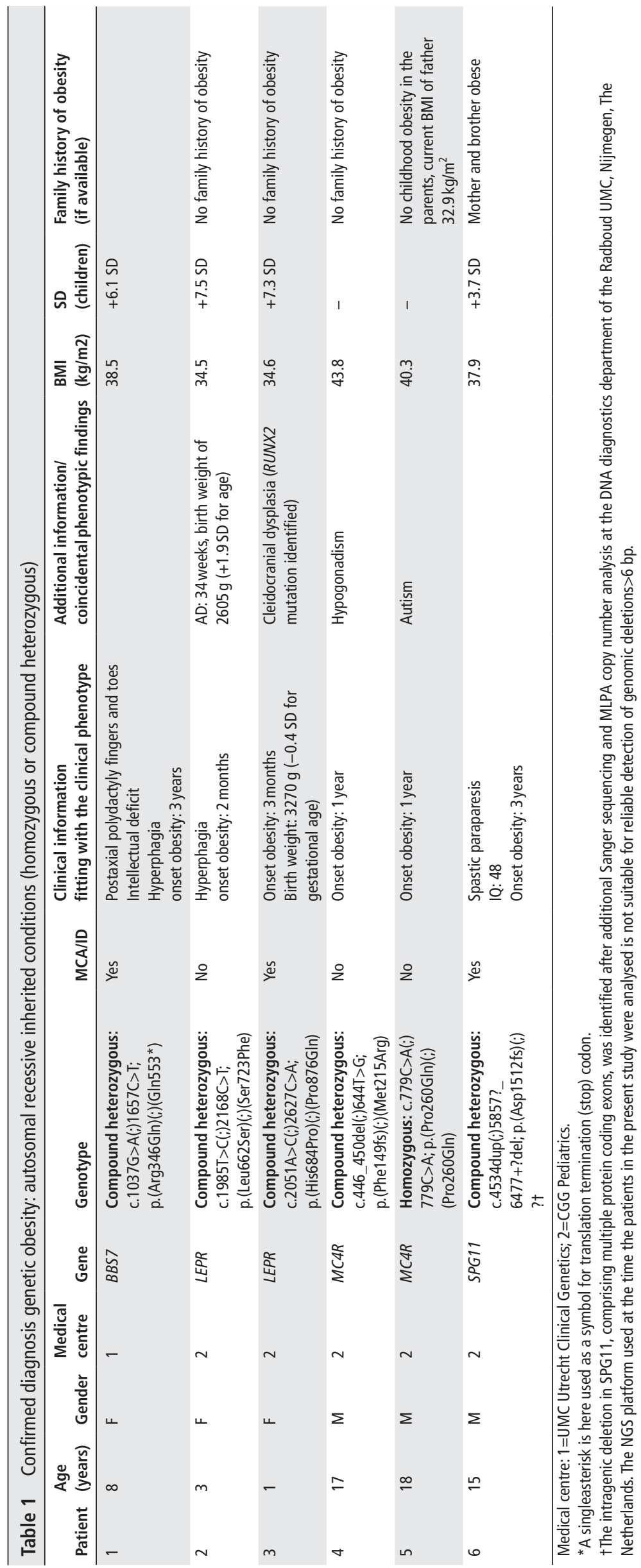

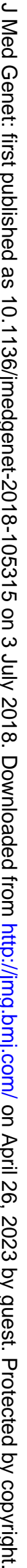




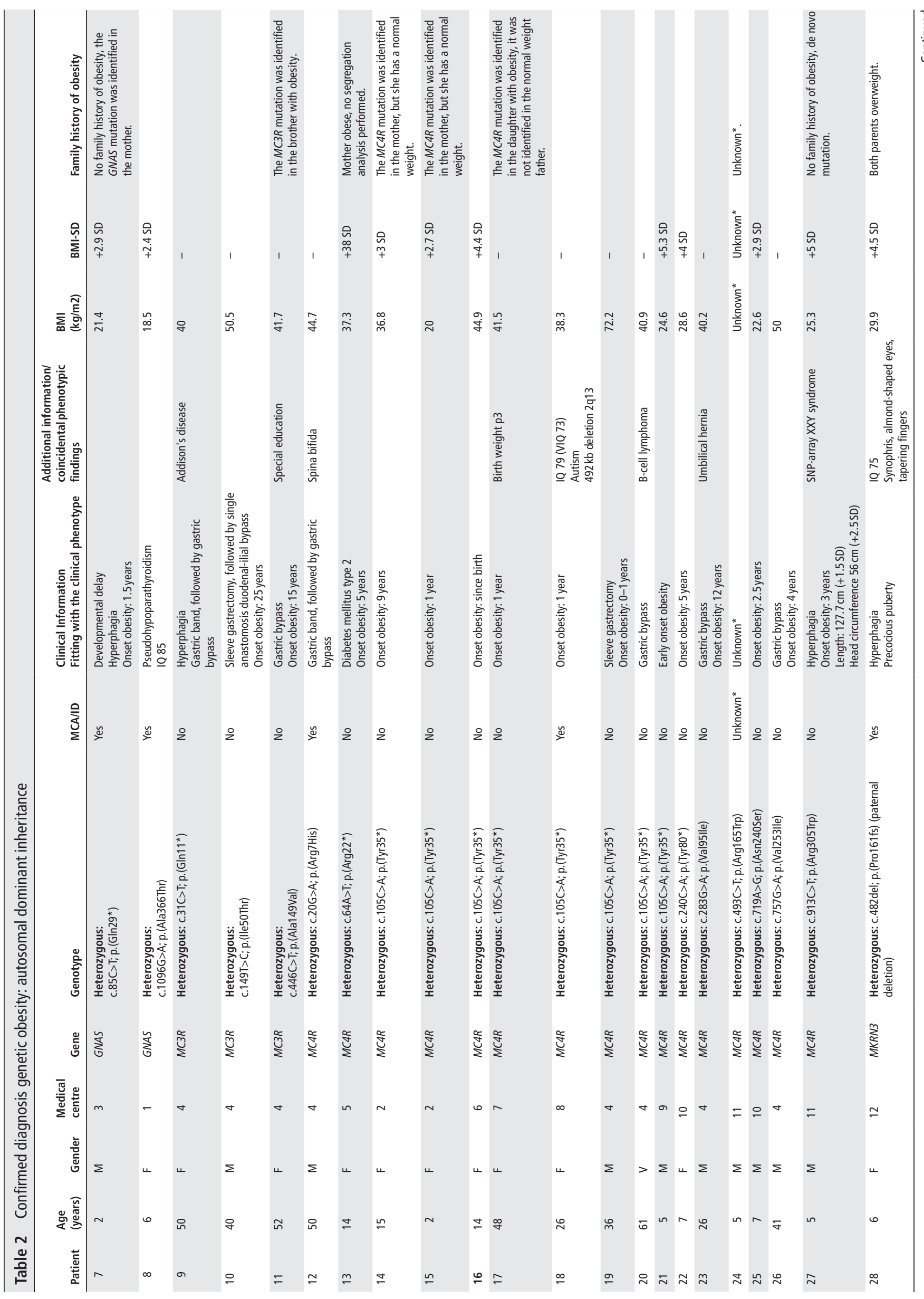




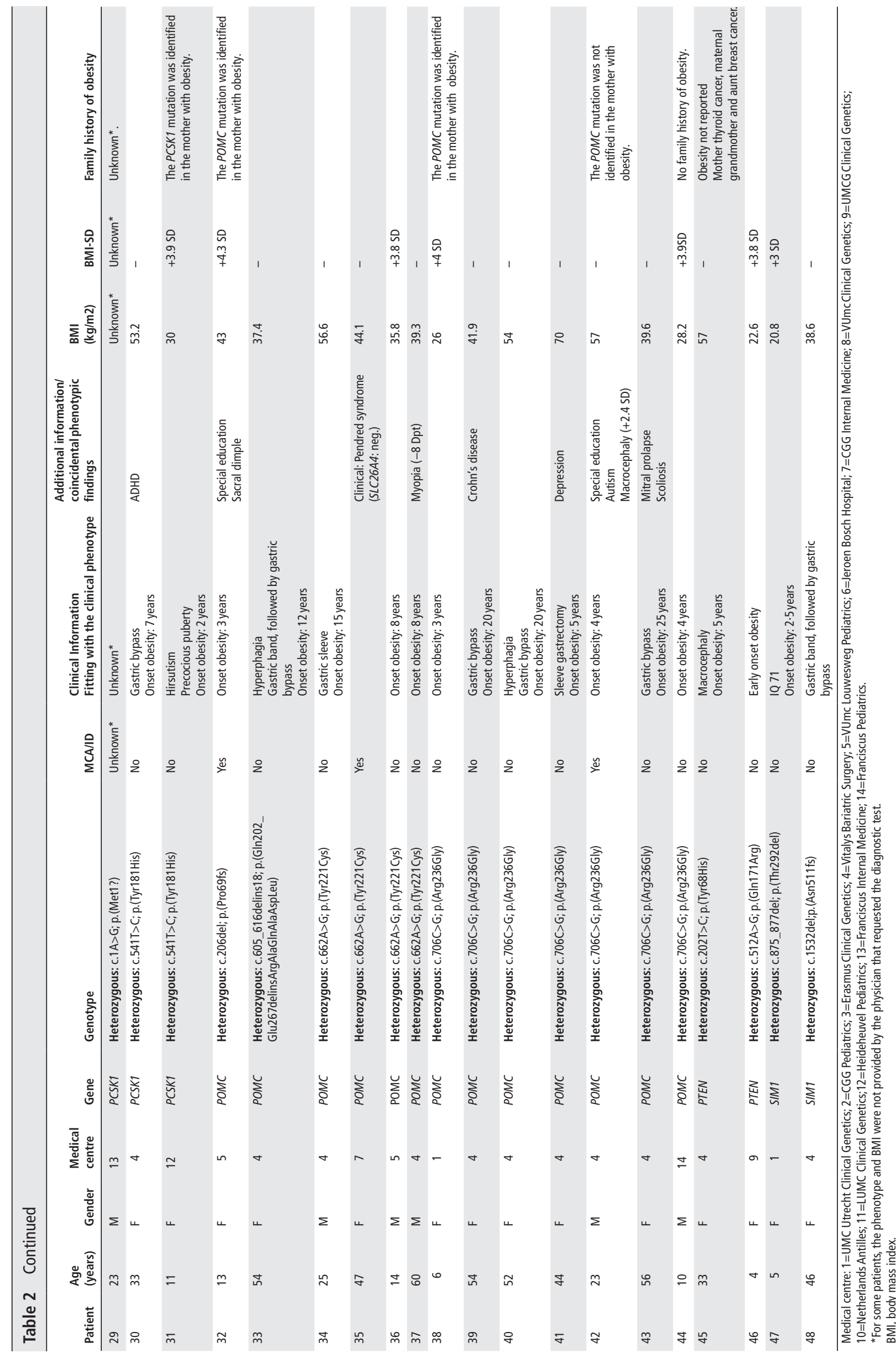

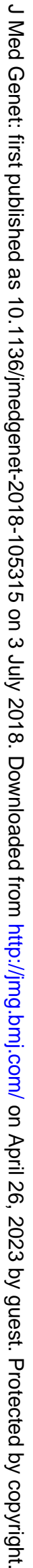


significant. Thus, the set of 27 curated pathogenic BBS mutations is not over-represented in the obesity gene panel cohort. This argues against a possible stronger predisposition to obesity for heterozygote $B B S$ gene mutation carriers compared with the other genes on the panel. Furthermore, we were able to perform segregation analysis in the family in 12 out of 48 patients with BBS-associated mutations. The identified mutation cosegregated with obesity in only 6 out of 12 cases (see online supplementary table S4).

\section{Illustrative cases and (future) personalised treatment Melanocortin-4 receptor}

Single pathogenic melanocortin-4 receptor (MC4R) mutations cause a hyperphagic phenotype resulting in obesity, which is milder than in patients with compound heterozygous or homozygous mutations. ${ }^{17}$ In our cohort, pathogenic MC4R mutations were identified in 18 patients (1.5\% of the total cohort), of which 16 patients were heterozygous for a MC4R mutation. The majority of these patients became obese before the age of 5 (see online supplementary table S5). Segregation analysis in families was performed in 9 of the 18 patients. Five out of nine patients showed cosegregation with the obesity phenotype. This result fits with the known variable penetrance. ${ }^{18}$ Four of the heterozygous MC4R patients were treated with a gastric bypass. Although (long-term) response treatment studies are pending, there is evidence that patients with heterozygous MC4R mutations have good results after bariatric surgery. ${ }^{19} 20$

In 8 of the 18 patients with MC4R mutations, we identified the same pathogenic mutation c. $105 \mathrm{C}>\mathrm{A}$; p.(Tyr35*). In all these patients, an additional c. $110 \mathrm{~A}>\mathrm{T}$; p.(Asp37Val) mutation was found in cis. The ExAC allele frequency of this mutation is $0.00004953 \%$; only present in the European (non-Finnish) population. This result is highly suggestive that the c.105C >A p. (Tyr35*) mutation is a European founder mutation.

\section{Leptin receptor}

Leptin receptor (LEPR) deficiency can cause obesity with hyperphagia, delayed pubertal development and immune problems. ${ }^{21}$ Patient 2 was diagnosed with a compound heterozygous leptin receptor deficiency. She was born at $33+6$ weeks of gestation with a birth weight of $2605 \mathrm{~g}$ (+1.9SD). The girl was severely hyperphagic since she was a few weeks old and became obese at the age of 2 months. At the age of 3 years, her BMI was $34.5 \mathrm{~kg} /$ $\mathrm{m}^{2}$ (+7.5 SD). In the first 4 months after the diagnosis, her BMI lowered to $30 \mathrm{~kg} / \mathrm{m}^{2}$ (+6SD). The identification of the LEPR mutations helped in the control of her weight due to supportive treatment. Treatment with setmelanotide, an MC4R agonist, might be a therapeutic option for patients with leptin receptor deficiency. ${ }^{10}$

\section{Proopiomelanocortin}

Homozygous and compound heterozygous proopiomelanocortin (POMC) mutations cause a combination of early onset obesity, ACTH deficiency, fair skin and red hair. ${ }^{22}$ Individuals heterozygous for $P O M C$ mutations are only predisposed to the obesity phenotype. ${ }^{23}$

We identified 13 patients with a heterozygous POMC mutation. One of these was a girl aged 6 years with a BMI of $26 \mathrm{~kg} / \mathrm{m}^{2}$ (+4SD). Besides hyperphagia, she had no physical or intellectual abnormalities. In this patient, the c.706C > G p.(Arg236Gly) mutation was identified, which was previously described in literature. ${ }^{24}$ Segregation analysis showed the same mutation in her mother with obesity.
This POMC mutation was also identified in an adult patient. She suffered from obesity since the age of 5 . At age 44 , she had a BMI of $70 \mathrm{~kg} / \mathrm{m}^{2}$. Besides hyperphagia and depression, she had no other abnormalities. A sleeve gastrectomy was recently performed. Long-term follow-up results are needed to assess the success of the operation.

Treatment with setmelanotide, an MC4R agonist, is a therapeutic option for patients with homozygous or compound heterozygous POMC mutations. ${ }^{10}$ Setmelanotide treatment might prove to be effective for heterozygous POMC patients as well.

\section{DISCUSSION}

Here, we present a large patient group for which diagnostic targeted NGS gene panel analysis of syndromic and non-syndromic obesity was performed (1230 affected individuals). A confirmed genetic diagnosis could be made in 48 of 1230 tested patients $(3.9 \%)$, with the highest yield in a paediatric subgroup 12/164 (7.3\%). In 67 additional patients, probable pathogenic mutations were found (5.4\%). Further segregation analysis or functional studies are needed to prove the pathogenicity of these mutations. Our data again confirm that obesity is a heterogeneous condition, with diagnoses made on the basis of mutations in at least 11 different genes. Other studies using an NGS approach in genetic obesity showed variable results: a study in Norway had a diagnostic yield of $0.8 \%$, only finding mutations in MC4R, whereas a study in Guadeloupean Afro-Caribbean children showed a yield of $>15 \% .^{725}$ From the 11 different genes in which mutations were found that lead to a definitive diagnosis in our cohort, MC4R mutations were the most frequent genetic cause of obesity. The results of our permutation analysis and segregation analysis argue against a possible stronger predisposition for obesity in heterozygote $B B S$ gene mutation carriers than the general population.

Some genetic causes of obesity such as CNVs (16p11.2 deletions), trinucleotide repeat expansion (fragile X-syndrome), uniparental disomies (UPD14) and methylation abnormalities (Prader-Willi syndrome) are not tested with the obesity gene panel. Because of the relatively high prevalence of $16 \mathrm{p} 11.2$ deletions as the cause of obesity and the variable phenotype of this syndrome, we would recommend to add SNP-array analysis to the diagnostic approach of a patient with suspected genetic obesity. This could result in a higher diagnostic yield than the definite molecular diagnosis of 3.9\% that we present here with NGS gene panel testing. Since research in obesity genetics is rapidly progressing, recently identified obesity-associated genes, such as CPE were not included in this panel. ${ }^{26}$ These genes can be added to the next version of our diagnostic obesity gene panel.

Six out of the 48 patients with a definitive diagnosis $(12.5 \%)$ had a mutation that causes a syndromic form of obesity. The majority of the identified mutations however, are linked to non-syndromic monogenic forms of obesity. This may be caused by inclusion bias: patients with a syndromic form of obesity might already have a genetic diagnosis for their developmental disorders or congenital anomalies that presented at earlier age than the obesity. The diagnostic yield of genetic testing in obesity is low in unselected populations, but can be increased by targeting it to patients with specific phenotypes. From the patient's perspective, it can be an important test because of personalised treatment and future treatment options. Promising drug trials for POMC and LEPR deficiency are currently being performed. ${ }^{10}$ An established diagnosis of genetic obesity might influence the choice for bariatric surgery as well. Short-term 
effects of bariatric surgery in patients with monogenic obesity (due to MC4R heterozygous mutations) seem to be comparable to patients without a genetic diagnosis, ${ }^{27}{ }^{28}$ but there are only a few reports in literature about long-term effects. Two single case reports on long-term effects of bariatric surgery describe significant weight regain in the years after bariatric surgery in patients with homozygous mutations in LEPR and MC4R, respectively. ${ }^{29}{ }^{30}$ We are still awaiting the long-term follow-up results for the bariatric subgroup in our cohort.

A limitation of this study is that we compare the variants with the ExAC database, which does not exclude persons with obesity, so it is possible that rare pathogenic variants causing early onset obesity are present in ExAC resulting in an underestimation of our positive results. Moreover, the ExAC control group does not share the exact same geographic or ethnic characteristics with our Dutch cohort, possibly disregarding the occurrence of founder mutations in these populations.

Using our obesity gene panel, we have found more carrier statuses than definite diagnoses: 61 patients $(5 \%)$ were carriers of a pathogenic mutation associated with recessive disease. However, to our opinion the importance of the diagnosis outweighs the downside of identifying carrier statuses, since finding the genetic cause of inherited obesity can have a significant clinical relevance. Genetic counselling can be provided (including information about risks for offspring to be affected with a severe recessive condition) and some patients are eligible for specific therapies. Single gene testing of the most common genetic causes would reduce the problem of finding unclear results or carrier statuses; however, the costs of multiple standalone Sanger sequencing tests are much higher than the costs of this multigene panel. Finally, it could also be possible that combinations of several VUS increase obesity risk (polygenic effect), but that was not the purpose of our study and thus not examined.

In conclusion, our NGS-based gene panel analysis in patients with obesity led to a definitive diagnosis of a genetic obesity disorder in 3.9\% of the patients (48/1230). In 67 additional patients (5.4\%), probable pathogenic mutations were found for which the causal role in the obesity phenotype has yet to be confirmed. The obesity gene panel showed the highest yield in a paediatric subgroup, establishing a definitive diagnosis in 12 out of 164 children with severe early onset obesity (7.3\%).

The NGS-based gene panel analysis in patients with obesity is a useful tool for diagnosing genetic obesity and can have serious impact on the treatment of patients. Therefore, we recommend testing in selected patients with early onset severe obesity.

\section{Author affiliations \\ 'Department of Clinical Genetics, Academic Medical Center, Amsterdam, The Netherlands \\ ${ }^{2}$ Department of Genetics, Universitair Medisch Centrum Utrecht, Utrecht, The Netherlands \\ ${ }^{3}$ Departmentof Bariatric Surgery, Rijnstate Hospital, Arnhem, The Netherlands ${ }^{4}$ Department of Internal Medicine, division of Endocrinology, Erasmus MC, University Medical Center Rotterdam, Rotterdam, The Netherlands \\ ${ }^{5}$ Childhood Obesity Center Heideheuvel, Merem, Hilversum, The Netherlands \\ ${ }^{6}$ Child Obesity Expert Centre Amsterdam, Women and Child Clinic, VU Medical Center (previously Deptartment of Pediatrics Slotervaartziekenhuis), Amsterdam, The Netherlands \\ ${ }^{7}$ Department of Clinical Genetics, VU Medical Center, Amsterdam, The Netherlands \\ ${ }^{8}$ Department of Pediatric Endocrinology, Sophia kinderziekenhuis Erasmus MC University Medical Center Rotterdam, Rotterdam, The Netherlands}

Collaborators Genetic Obesity Consortium: FJ Berends; EO Aarts; S Bouma-de Jongh; I von Rosenstiel; EGAH van Mil; CJ De Groot; HE Veenstra-Knol; JBGM Verheij; KE Stuurman; VL Wester; YMC Hendriks; M Bakker.
Contributors $\mathrm{LK}, \mathrm{MM}, \mathrm{GvH}, \mathrm{BvdZ}$ and $\mathrm{MMvH}$ contributed to the study design and acquisition and/or analysis of the data. All authors contributed to the data interpretation, provided critical revisions of the manuscript and approved the final version.

Funding The authors have not declared a specific grant for this research from any funding agency in the public, commercial or not-for-profit sectors.

Disclaimer All authors, external and internal, had full access to all of the data (including statistical reports and tables) in the study and can take responsibility for the integrity of the data and the accuracy of the data analysis. We attest that we have obtained appropriate permissions. Required fees for use of copyright-protected materials: not applicable.

Competing interests None declared.

Patient consent Not required.

Provenance and peer review Not commissioned; externally peer reviewed.

Data sharing statement Extra clinical information might be available for some patients. Data that underlie the results reported in this article (after deidentification) can be requested if relevant. Proposals should be directed to $\mathrm{m}$. vanhaelst@vumc.n!.

\section{REFERENCES}

1 World Health Organization Fact Sheet 'Obesity and overweight'. 2017. http://www. who.int/mediacentre/factsheets/fs311/en/ (accessed 16 Jan 2018)

2 Domecq JP, Prutsky G, Leppin A, Sonbol MB, Altayar O, Undavalli C, Wang Z, Elraiyah $\mathrm{T}$, Brito JP, Mauck KF, et al. Clinical review: Drugs commonly associated with weight change: a systematic review and meta-analysis. J Clin Endocrinol Metab 2015;100:363-70.

3 Stryjecki C, Alyass A, Meyre D. Ethnic and population differences in the genetic predisposition to human obesity. Obes Rev 2018;19:62-80.

4 Yazdi FT, Clee SM, Meyre D. Obesity genetics in mouse and human: back and forth, and back again. PeerJ 2015;3:e856.

5 Faroogi S, O'Rahilly S. Genetics of obesity in humans. Endocr Rev 2006;27:710-8. Obesity (Silver Spring). 2015;23:1687-95.

6 Saeed S, Bonnefond A, Manzoor J, Shabbir F, Ayesha H, Philippe J, Durand E, Crouch H Sand $\mathrm{O}$, Ali M, et al. Genetic variants in LEP, LEPR, and MC4R explain $30 \%$ of severe obesity in children from a consanguineous population. Obesity 2015;23:1687-95.

7 Foucan L, Larifla L, Durand E, Rambhojan C, Armand C, Michel CT, Billy R, Dhennin $V$, De Graeve F, Rabearivelo I, et al. High Prevalence of Rare Monogenic Forms of Obesity in Obese Guadeloupean Afro-Caribbean Children. J Clin Endocrinol Metab 2018;103:539-545

8 Ramachandrappa S, Farooqi IS. Genetic approaches to understanding human obesity. J Clin Invest 2011:121:2080-6.

9 Faroogi IS, Jebb SA, Langmack G, Lawrence E, Cheetham CH, Prentice AM, Hughes IA, McCamish MA, O'Rahilly S. Effects of recombinant leptin therapy in a child with congenital leptin deficiency. N Eng/ J Med 1999;341:879-84.

10 Kühnen P, Clément K, Wiegand S, Blankenstein O, Gottesdiener K, Martini LL, Mai K, Blume-Peytavi U, Grüters A, Krude H. Proopiomelanocortin Deficiency Treated with a Melanocortin-4 Receptor Agonist. N Engl J Med 2016;375:240-6.

11 Collet TH, Dubern B, Mokrosinski J, Connors H, Keogh JM, Mendes de Oliveira E, Henning E, Poitou-Bernert C, Oppert JM, Tounian P, et al. Evaluation of a melanocortin-4 receptor (MC4R) agonist (Setmelanotide) in MC4R deficiency. Mol Metab 2017:6:1321-9.

12 Wee CC, Davis RB, Huskey KW, Jones DB, Hamel MB. Quality of life among obese patients seeking weight loss surgery: the importance of obesity-related social stigma and functional status. J Gen Intern Med 2013;28:231-8.

13 Pigeyre M, Yazdi FT, Kaur Y, Meyre D. Recent progress in genetics, epigenetics and metagenomics unveils the pathophysiology of human obesity. Clin Sci 2016;130:943-86.

14 Faroogi IS, O'Rahilly $\mathrm{S}$. New advances in the genetics of early onset obesity. Int J Obes 2005:29:1149-52.

15 Croft JB, Morrell D, Chase CL, Swift M. Obesity in heterozygous carriers of the gene for the Bardet-Biedl syndrome. Am J Med Genet 1995;55:12-15.

16 Beales PL, Elcioglu N, Woolf AS, Parker D, Flinter FA. New criteria for improved diagnosis of Bardet-Biedl syndrome: results of a population survey. J Med Genet 1999:36:437-46

17 Faroogi IS, Yeo GS, Keogh JM, Aminian S, Jebb SA, Butler G, Cheetham T, O'Rahilly S. Dominant and recessive inheritance of morbid obesity associated with melanocortin 4 receptor deficiency. J Clin Invest 2000;106:271-9.

18 Dubern B, Clément K, Pelloux V, Froguel P, Girardet JP, Guy-Grand B, Tounian P. Mutational analysis of melanocortin-4 receptor, agouti-related protein, and alpha-melanocyte-stimulating hormone genes in severely obese children. J Pediatr 2001;139:204-9.

19 Jelin EB, Daggag H, Speer AL, Hameed N, Lessan N, Barakat M, Nadler EP. Melanocortin-4 receptor signaling is not required for short-term weight loss after sleeve gastrectomy in pediatric patients. Int J Obes 2016;40:550-3. 
20 Aslan IR, Campos GM, Calton MA, Evans DS, Merriman RB, Vaisse C. Weight Loss after Roux-en-Y Gastric Bypass in Obese Patients Heterozygous for MC4R Mutations. Obes Surg 2011;21:930-4.

21 Clément K, Vaisse C, Lahlou N, Cabrol S, Pelloux V, Cassuto D, Gourmelen M, Dina C, Chambaz J, Lacorte JM, et al. A mutation in the human leptin receptor gene causes obesity and pituitary dysfunction. Nature 1998;392:398-401.

22 Krude H, Biebermann H, Luck W, Horn R, Brabant G, Grüters A. Severe early-onset obesity, adrenal insufficiency and red hair pigmentation caused by POMC mutations in humans. Nat Genet 1998;19:155-7.

23 Farooqi IS, Drop S, Clements A, Keogh JM, Biernacka J, Lowenbein S, Challis BG, O'Rahilly S. Heterozygosity for a POMC-null mutation and increased obesity risk in humans. Diabetes 2006;55:2549-53.

24 Challis BG, Pritchard LE, Creemers JW, Delplanque J, Keogh JM, Luan J, Wareham NJ, Yeo GS, Bhattacharyya S, Froguel P, et al. A missense mutation disrupting a dibasic prohormone processing site in pro-opiomelanocortin (POMC) increases susceptibility to early-onset obesity through a novel molecular mechanism. Hum Mol Genet 2002;11:1997-2004.

25 Nordang GBN, Busk ØL, Tveten K, Hanevik HI, Fell AKM, Hjelmesæth J, Holla ØL, Hertel JK. Next-generation sequencing of the monogenic obesity genes LEP, LEPR,
MC4R, PCSK1 and POMC in a Norwegian cohort of patients with morbid obesity and normal weight controls. Mol Genet Metab 2017;121:51-6.

26 Alsters SI, Goldstone AP, Buxton JL, Zekavati A, Sosinsky A, Yiorkas AM, Holder S, Klaber RE, Bridges N, van Haelst MM, et al. Truncating Homozygous Mutation of Carboxypeptidase E (CPE) in a Morbidly Obese Female with Type 2 Diabetes Mellitus, Intellectual Disability and Hypogonadotrophic Hypogonadism. PLoS One 2015; 10:e0131417.

27 Valette M, Poitou C, Le Beyec J, Bouillot JL, Clement K, Czernichow S. Melanocortin-4 receptor mutations and polymorphisms do not affect weight loss after bariatric surgery. PLoS One 2012;7:e48221.

28 Aslan IR, Campos GM, Calton MA, Evans DS, Merriman RB, Vaisse C. Weight loss after Roux-en-Y gastric bypass in obese patients heterozygous for MC4R mutations. Obes Surg 2011;21:930-4.

29 Le Beyec J, Cugnet-Anceau C, Pépin D, Alili R, Cotillard A, Lacorte JM, Basdevant $A$, Laville $M$, Clément $K$. Homozygous leptin receptor mutation due to uniparenta disomy of chromosome 1: response to bariatric surgery. J Clin Endocrinol Metab 2013;98:E397-E402.

30 Aslan IR, Ranadive SA, Ersoy BA, Rogers SJ, Lustig RH, Vaisse C. Bariatric surgery in a patient with complete MC4R deficiency. Int J Obes 2011;35:457-61. 